\title{
Construction of Intuitionistic Fuzzy Mappings with Applications
}

\author{
Soheyb Milles ${ }^{1 *}$, Ergün Nart $^{2}$, Farhan Ismail $^{2}$ and Abdelkrim Latreche ${ }^{3}$ \\ ${ }^{1}$ Laboratory of Pure and Applied Mathematics, Department of Mathematics, University of M'sila, Algeria \\ ${ }^{2}$ Department of Mechatronics Engineering, Faculty of Technology, Sakarya University of Applied Sciences, Sakarya, Turkey \\ ${ }^{3}$ Department of Technology, Faculty of Technology, 20 Août 1955 University, Skikda, Algeria \\ * Corresponding author
}

\section{Article Info}

Keywords: Intuitionistic fuzzy set, Intuitionistic fuzzy mapping, Intuitionistic fuzzy topology

2010 AMS: 03E72, 58C07, 54A40.

Received: 29 May 2020

Accepted: 29 September 2020

Available online: 23 December 2020

\begin{abstract}
In a recent paper, Ismail and Massa'deh have introduced the notion of L-fuzzy mapping and some basic operations were proved. In this paper, we generalize this notion to the setting of intuitionistic fuzzy sets. Moreover, we study the main properties related to intuitionistic fuzzy mapping. As applications, we provide properties of intuitionistic fuzzy continuous mappings in intuitionistic fuzzy topological spaces and investigate the relation among various kinds of intuitionistic fuzzy continuity.
\end{abstract}

\section{Introduction}

Mappings in crisp set theory are very well known and play a prominent role in mathematical branches such as topology and its analysis approaches. They appear to enhance the concept of functional predicate in formal logic [14] and also closely related to category theory [23]. In dynamical systems, a mapping denotes an evolution function used to create discrete dynamical systems [11].

In fuzzy setting, several authors introduce and investigate the concept of fuzzy mapping in different ways. Heilpern [13] introduced the concept of fuzzy mapping and proved a fixed point theorem for fuzzy contraction mappings. Ismail and Massa'deh [10] defined L-fuzzy mappings and studied their operations, also they developed many properties of classical mappings into L-fuzzy case. Lim et al. [19] investigated the equivalence relations and mappings for fuzzy sets and relationship among them.

In 1983, Atanassov [1] introduced the concept of intuitionistic fuzzy set which is a generalization of Zadeh's fuzzy set previously introduced in [24] by using two membership functions for the elements of the universe of discourse. After that, several intuitionistic fuzzy concepts are studied by many authors. For the concept of mapping, an extended approaches are proposed based on Atanassov's intuitionistic fuzzy sets. Kang et al. [18] introduced the concept of intuitionistic fuzzy mapping and they give the decomposition of an intuitionistic fuzzy mapping by using intuitionistic fuzzy equivalence relations. Shen et al. [22] presented the notion of intuitionistic fuzzy mapping as a generalization of fuzzy mapping, and they established the decomposition and representation theorems of intuitionistic fuzzy mappings. Very recently, Gomathi and Jayanthi [12] introduced the concept of intuitionistic fuzzy $b^{\sharp}$ continuous mapping in intuitionistic fuzzy topological spaces and discussed some of their properties and characterizations. For more details about intuitionistic fuzzy mappings and background, the readers are referred to $[16,20,25]$ and more others.

In this paper, we continue further by generalizing the notion of fuzzy mapping introduced by Ismail and Massa'deh to the intuitionistic fuzzy setting. Hereafter, the main properties related to intuitionistic fuzzy mapping are studied. Also, we generalize the notion of fuzzy topology on fuzzy sets to the intuitionistic fuzzy case to provide properties of intuitionistic fuzzy continuous mappings. To that end, the relations among intuitionistic fuzzy continuity, precontinuity and $\alpha$-continuity are investigated.

This paper is structured as follows. After recalling some basic definitions and properties in Section 2, the notion of intuitionistic fuzzy mapping by construction on a set is introduced, and some basic properties are given in Section 3. As applications, some properties of intuitionistic fuzzy continuous mappings in intuitionistic fuzzy topological space are provided and relations among some kinds of intuitionistic fuzzy continuity in Section 4 are investigated. Finally, some conclusions and future research in Section 5 are presented. 


\section{Preliminaries}

This section contains the basic definitions and properties of intuitionistic fuzzy sets, intuitionistic fuzzy relations and some related notions that will be needed throughout this paper.

\subsection{Atanassov's intuitionistic fuzzy sets}

In this subsection we recall some basic concepts of intuitionistic fuzzy sets.

Let $X$ be a universe, then a fuzzy set $A=\left\{\left\langle x, \mu_{A}(x)\right\rangle \mid x \in X\right\}$ defined by Zadeh [24] is characterized by a membership function $\mu_{A}: X \rightarrow[0,1]$, where $\mu_{A}(x)$ is interpreted as the degree of a membership of the element $x$ in the fuzzy subset $A$ for each $x \in X$.

Atanassov in [1] introduced another fuzzy object, called intuitionistic fuzzy set as a generalization of the concept of fuzzy set, shown as follows

$$
A=\left\{\left\langle x, \mu_{A}(x), v_{A}(x)\right\rangle \mid x \in X\right\},
$$

which is characterized by a membership function $\mu_{A}: X \rightarrow[0,1]$ and a non-membership function $v_{A}: X \rightarrow[0,1]$, with the condition

$$
0 \leq \mu_{A}(x)+v_{A}(x) \leq 1
$$

for any $x \in X$. The numbers $\mu_{A}(x)$ and $v_{A}(x)$ represent, respectively, the membership degree and the non-membership degree of the element $x$ in the intuitionistic fuzzy set $A$ for each $x \in X$.

In the fuzzy set theory, the non-membership degree of an element $x$ of the universe is defined as $v_{A}(x)=1-\mu_{A}(x)$ (using the standard negation) and thus it is fixed. In intuitionistic fuzzy setting, the non-membership degree is a more-or-less independent degree: the only condition is that $v_{A}(x) \leq 1-\mu_{A}(x)$. Certainly fuzzy sets are intuitionistic fuzzy sets by setting $v_{A}(x)=1-\mu_{A}(x)$, but not conversely.

Throughout this paper, authors denote the set of all intuitionistic fuzzy sets in a set $X$ as $\operatorname{IFS}(X)$ and $X, Y, Z, \ldots$ etc., will be nonempty crisp sets.

Definition 2.1. [1] Let $A=\left\{\left\langle x, \mu_{A}(x), v_{A}(x)\right\rangle \mid x \in X\right\}$ and $B=\left\{\left\langle x, \mu_{B}(x), v_{B}(x)\right\rangle \mid x \in X\right\}$, be two IFSs on a set $X$. Then

(i) $A \subseteq B$ if $\mu_{A}(x) \leq \mu_{B}(x)$ and $v_{A}(x) \geq v_{B}(x)$, for all $x \in X$,

(ii) $A=B$ if $\mu_{A}(x)=\mu_{B}(x)$ and $v_{A}(x)=v_{B}(x)$, for all $x \in X$,

(iii) $A \cap B=\left\{\left\langle x, \mu_{A}(x) \wedge \mu_{B}(x), v_{A}(x) \vee v_{B}(x)\right\rangle \mid x \in X\right\}$

(iv) $A \cup B=\left\{\left\langle x, \mu_{A}(x) \vee \mu_{B}(x), v_{A}(x) \wedge v_{B}(x)\right\rangle \mid x \in X\right\}$,

(v) $\bar{A}=\left\{\left\langle x, v_{A}(x), \mu_{A}(x)\right\rangle \mid x \in X\right\}$,

(vi) $[A]=\left\{\left\langle x, \mu_{A}(x), 1-\mu_{A}(x)\right\rangle \mid x \in X\right\}$,

(vii) $\langle A\rangle=\left\{\left\langle x, 1-v_{A}(x), v_{A}(x)\right\rangle \mid x \in X\right\}$.

For more details please refer to $([1-3,21,25])$.

Definition 2.2. [3] Let A be an intuitionistic fuzzy set on universe $X$. The support of $A$ is the crisp subset of $X$ given by

$$
\operatorname{Supp}(A)=\left\{x \in X \mid \mu_{A}(x)>0 \text { or }\left(\mu_{A}(x)=0 \text { and } v_{A}(x)<1\right)\right\} .
$$

In the sequel, we need the following definition of level set (which is also often called $(\alpha, \beta)$-cut) of intuitionistic fuzzy set.

Definition 2.3. [15] Let $A$ be an intuitionistic fuzzy set on a nonempty set $X$. The $(\alpha, \beta)$-cut of $A$ is the crisp subset

$$
A_{(\alpha, \beta)}=\left\{x \in X \mid \mu_{A}(x) \geq \alpha \text { and } v_{A}(x) \leq \beta\right\}
$$

where $\alpha, \beta \in[0,1]$ with $\alpha+\beta \leq 1$.

\subsection{Intuitionistic fuzzy relations}

Burillo and Bustince [4,5] introduced the concept of intuitionistic fuzzy relation as a natural generalization of fuzzy relation.

Definition 2.4. [4,5] An intuitionistic fuzzy binary relation (An intuitionistic fuzzy relation, for short) from a universe $X$ to a universe $Y$ is an intuitionistic fuzzy subset in $X \times Y$, i.e., is an expression $R$ given by

$$
R=\left\{\left\langle(x, y), \mu_{R}(x, y), v_{R}(x, y)\right\rangle \mid(x, y) \in X \times Y\right\},
$$

where

$$
\mu_{R}: X \times Y \rightarrow[0,1] \text {, and } v_{A}: X \times Y \rightarrow[0,1]
$$

satisfy the condition

$$
0 \leq \mu_{R}(x, y)+v_{R}(x, y) \leq 1
$$

for any $(x, y) \in X \times Y$. The value $\mu_{R}(x, y)$ is called the degree of a membership of $(x, y)$ in $R$ and $v_{R}(x, y)$ is called the degree of a non-membership of $(x, y)$ in $R$. 
Next, the following definitions is needed to recall.

Definition 2.5. Let $R$ and $P$ be two intuitionistic fuzzy relations from a universe $X$ to a universe $Y$.

(i) The transpose (inverse) $R^{t}$ of $R$ is the intuitionistic fuzzy relation from the universe $Y$ to the universe $X$ defined by

$$
R^{t}=\left\{\left\langle(x, y), \mu_{R^{t}}(x, y), v_{R^{t}}(x, y)\right\rangle \mid(x, y) \in X \times Y\right\},
$$

where

$$
\left\{\begin{array}{c}
\mu_{R^{t}}(x, y)=\mu_{R}(y, x) \\
\text { and } \\
v_{R^{t}}(x, y)=v_{R}(y, x)
\end{array}\right.
$$

for any $(x, y) \in X \times Y$.

(ii) $R$ is said to be contained in $P$ or we say that $P$ contains $R$, denoted by $R \subseteq P$, iffor all $(x, y) \in X \times Y$ it holds that $\mu_{R}(x, y) \leq \mu_{P}(x, y)$ and $v_{R}(x, y) \geq v_{P}(x, y)$.

(iii) The intersection (resp. the union) of two intuitionistic fuzzy relations $R$ and $P$ from a universe $X$ to a universe $Y$ is an intuitionistic fuzzy relation defined as

$$
R \cap P=\left\{\left\langle(x, y), \min \left(\mu_{R}(x, y), \mu_{P}(x, y)\right), \max \left(v_{R}(x, y), v_{P}(x, y)\right)\right\rangle \mid(x, y) \in X \times Y\right\}
$$

and

$$
R \cup P=\left\{\left\langle(x, y), \max \left(\mu_{R}(x, y), \mu_{P}(x, y)\right), \min \left(v_{R}(x, y), v_{P}(x, y)\right)\right\rangle \mid(x, y) \in X \times Y\right\} .
$$

The following properties are crucial in this paper (see e.g. $[4,5,8]$ ).

Definition 2.6. Let $R$ be an intuitionistic fuzzy relation from a universe $X$ into itself.

(i) Reflexivity: $\mu_{R}(x, x)=1$, for any $x \in X$. In this case we note that $v_{R}(x, x)=0$, for any $x \in X$.

(ii) Antisymmetry: for any $x, y \in X, x \neq y$ then

$$
\left\{\begin{array}{c}
\mu_{R}(x, y) \neq \mu_{R}(y, x) \\
v_{R}(x, y) \neq v_{R}(y, x) \\
\pi_{R}(x, y)=\pi_{R}(y, x)
\end{array}\right.
$$

where $\pi_{R}(x, y)=1-\mu_{R}(x, y)-v_{R}(x, y)$.

(iii) Perfect antisymmetry: for any $x, y \in X$ with $x \neq y$ and

$$
\left\{\begin{array}{l}
\mu_{R}(x, y)>0 \\
\text { or } \\
\mu_{R}(x, y)=0 \text { and } v_{R}(x, y)<1,
\end{array}\right.
$$

then

$$
\left\{\begin{array}{c}
\mu_{R}(y, x)=0 \\
\text { and } \\
v_{R}(y, x)=1
\end{array}\right.
$$

(iv) Transitivity: $R \supseteq R \circ_{\lambda, \rho}^{\alpha, \beta} R$.

In the above definition, the composition $R \circ_{\lambda, \rho}^{\alpha, \beta} R$ used in the transitivity means that

$$
R{ }_{\lambda, \rho}^{\alpha, \beta} R=\left\{\left\langle(x, z), \alpha_{y \in X}\left\{\beta\left[\mu_{R}(x, y), \mu_{R}(y, z)\right]\right\}, \lambda_{y \in X}\left\{\rho\left[v_{R}(x, y), v_{R}(y, z)\right]\right\}\right\rangle \mid x, z \in X\right\},
$$

where $\alpha, \beta, \lambda$ and $\rho$ are t-norms or t-conorms taken under the intuitionistic fuzzy condition

$$
0 \leq \alpha_{y \in X}\left\{\beta\left[\mu_{R}(x, y), \mu_{R}(y, z)\right]\right\}+\lambda_{y \in X}\left\{\rho\left[v_{R}(x, y), v_{R}(y, z)\right]\right\} \leq 1,
$$

for any $x, z \in X$.

The properties of this composition and the choice of $\alpha, \beta, \lambda$ and $\rho$, for which this composition fulfills a maximal number of properties, are investigated in [4]- [8]. 


\section{Construction of intuitionistics fuzzy mappings}

In crisp set theory, mappings are defined as binary relations. In this section, the notion of intuitionistic fuzzy mapping as intuitionistic fuzzy relations by construction on a set is introduced, and some basic properties are given.

Definition 3.1. Let $A$ be an intuitionistic fuzzy set on $X$ and $B$ be an intuitionistic fuzzy set on $Y$, let $f:$ Supp $A \rightarrow$ Supp B be an ordinary mapping and $R$ be an intuitionistic fuzzy relation on $X \times Y$. Then $f_{R}$ is called an intuitionistic fuzzy mapping iffor all $(x, y) \in \operatorname{Supp} A \times \operatorname{Supp} B$ the following condition is satisfied:

$$
\mu_{R}(x, y)=\left\{\begin{array}{c}
\min \left(\mu_{A}(x), \mu_{B}(f(x)), \text { if } y=f(x)\right. \\
0, \text { Otherwise }
\end{array}\right.
$$

and

$$
v_{R}(x, y)=\left\{\begin{array}{c}
\max \left(v_{A}(x), v_{B}(f(x)), \text { if } y=f(x)\right. \\
1, \text { Otherwise }
\end{array}\right.
$$

with $0 \leq \mu_{R}(x, y)+v_{R}(x, y) \leq 1$

Example 3.2. Let $X=\{\alpha, \beta\}, Y=\{1,2,3\}, A \in \operatorname{IFS}(X)$ and $B \in \operatorname{IFS}(Y)$ given by :

$$
A=\{\langle\alpha, 0.5,0.2\rangle,\langle\beta, 0.1,0.7\rangle\} \text { and } B=\{\langle 1,0,1\rangle,\langle 2,0.1,0.5\rangle,\langle 3,0.7,0.2\rangle\}
$$

We will construct the intuitionistic fuzzy mapping $f_{R}$ by :

(i) an ordinary mapping $f:\{\alpha, \beta\} \rightarrow\{2,3\}$ such that $f(\alpha)=2$ and $f(\beta)=3$,

(ii) an intuitionistic fuzzy relation $R$ defined by:

$$
\begin{aligned}
& \mu_{R}(\alpha, f(\alpha))=\mu_{R}(\alpha, 2)=\mu_{A}(\alpha) \wedge \mu_{B}(2)=0.1 \\
& \mu_{R}(\beta, f(\beta))=\mu_{R}(\beta, 3)=\mu_{A}(\beta) \wedge \mu_{B}(3)=0.1 \\
& \mu_{R}(\alpha, 1)=\mu_{R}(\alpha, 3)=\mu_{R}(\beta, 1)=\mu_{R}(\beta, 2)=0 \\
& \text { In similar way, it holds that } \\
& v_{R}(\alpha, f(\alpha))=v_{R}(\alpha, 2)=v_{A}(\alpha) \vee v_{B}(2)=0.5 \\
& v_{R}(\beta, f(\beta))=v_{R}(\beta, 3)=v_{A}(\beta) \vee v_{B}(3)=0.7 \\
& v_{R}(\alpha, 1)=v_{R}(\alpha, 3)=v_{R_{I}}(\beta, 1)=v_{R_{I}}(\beta, 2)=1 . \\
& \text { Hence, } \mu_{R}(x, y)=\{\langle(\alpha, f(\alpha)), 0.1,0.5\rangle,\langle(\beta, f(\beta)), 0.1,0.7\rangle,\langle(\alpha, 1), 0,1\rangle, \\
& \langle(\alpha, 3), 0,1\rangle,\langle(\beta, 1), 0,1\rangle,\langle(\beta, 2), 0,1\rangle\} .
\end{aligned}
$$

Thus, $f_{R}$ is an intuitionistic fuzzy mapping.

Remark 3.3. From the above definition, we can construct the intuitionistic fuzzy mapping by this method

(i) We determine the Supp A and Supp B.

(ii) We determine the ordinary mapping from Supp A to Supp B.

(iii) We determine the intuitionistic fuzzy relation $R$ to get the relationship degree and non-relationship degree between each element and its image.

(iv) Finally, we conclude the construction of the intuitionistic fuzzy mapping.

Definition 3.4. Let $f_{R}, g_{S}$ be two intuitionistic fuzzy mappings, then $f_{R}$ and $g_{S}$ are equal if and only if $f=g$ and $R=S$ i.e., $\left(\mu_{R}(x, f(x))=\right.$ $\mu_{S}(x, g(x))$ and $\left.v_{R}(x, f(x))=v_{S}(x, g(x))\right)$.

Definition 3.5. Let $A$ be an intuitionistic fuzzy set on $X$, let $f:$ Supp $A \rightarrow$ Supp A be an ordinary mapping such that $f(x)=x$ and $R$ be an intuitionistic fuzzy relation on $X \times X$. Then $f_{R}$ is called an intuitionistic fuzzy identity mapping if for all $x, y \in$ Supp A the following condition is satisfied:

$$
\mu_{R}(x, y)=\left\{\begin{array}{c}
\mu_{A}(x), \text { if } x=y \\
0, \text { Otherwise }
\end{array}\right.
$$

and

$$
v_{R}(x, y)=\left\{\begin{array}{c}
v_{A}(x), \text { if } x=y \\
1, \text { Otherwise }
\end{array}\right.
$$

with $0 \leq \mu_{R}(x, y)+v_{R}(x, y) \leq 1$.

Definition 3.6. Let $A, B$ and $C$ are an intuitionistic fuzzy sets on $X, Y$ and $Z$ respectively, let $f:$ Supp $A \rightarrow$ Supp B and $g:$ Supp B $\rightarrow$ Supp $C$ are an ordinary mappings and $R, S$ are an intuitionistic fuzzy relations on $X \times Y$ and $Y \times Z$ respectively. Then $(g \circ f)_{T}$ is called the composition of intuitionistic fuzzy mappings $f_{R}$ and $g_{R}$ such that $g \circ f:$ Supp $A \rightarrow$ Supp C and the intuitionistic fuzzy relation $T$ is defined by

$$
\left\{\begin{array}{c}
\mu_{T}(x, z)=\sup _{y}\left(\min \left(\mu_{R}(x, y), \mu_{S}(y, z)\right)\right) \\
\text { and } \\
v_{T}(x, z)=\inf _{y}\left(\max \left(v_{R}(x, y), v_{S}(y, z)\right)\right),
\end{array}\right.
$$

for any $(x, z) \in \operatorname{Supp} A \times \operatorname{Supp} C$. 
Remark 3.7. The intuitionistic fuzzy relation $T$ in the above definition can be written as follows: $\mu_{T}(x, z)=\min \left\{\mu_{A}(x), \mu_{B}(f(x)), \mu_{C}(g(f(x))\}\right.$ and $v_{T}(x, z)=\max \left\{v_{A}(x), v_{B}(f(x)), v_{C}(g(f(x))\}\right.$. Indeed, for any $(x, z) \in \operatorname{Supp} A \times \operatorname{Supp} C$, we have

$$
\begin{aligned}
\mu_{T}(x, z) & =\mu_{T}(x, g(f(x))) \\
& =\mu_{S \circ R}(x, g(f(x))) \\
& =\sup _{y}\left\{\operatorname { m i n } \left\{\mu_{R}(x, y), \mu_{S}(y, g(f(x)))\right.\right. \\
& \left.\left.=\min \left\{\mu_{R}(x, f(x))\right), \mu_{S}(f(x)), g(f(x))\right)\right\} \\
& =\min \left\{\mu_{A}(x), \mu_{B}(f(x)), \mu_{C}(g(f(x))\} .\right.
\end{aligned}
$$

Similarly, for any $(x, z) \in \operatorname{Supp} A \times \operatorname{Supp} C$, it holds that

$$
\begin{aligned}
v_{T}(x, z) & =v_{T}(x, g(f(x))) \\
& =v_{S \circ R}(x, g(f(x))) \\
& =\inf _{y}\left\{\operatorname { m a x } \left\{v_{R}(x, y), v_{S}(y, g(f(x)))\right.\right. \\
& \left.\left.=\max \left\{v_{R}(x, f(x))\right), v_{S}(f(x)), g(f(x))\right)\right\} \\
& =\max \left\{v_{A}(x), v_{B}(f(x)), v_{C}(g(f(x))\} .\right.
\end{aligned}
$$

Example 3.8. Let $X=\mathbb{N}, Y=\mathbb{R}$ and $Z=\mathbb{R}$, and let $A \in \operatorname{IFS}(X), B \in \operatorname{IFS}(Y)$ and $C \in \operatorname{IFS}(Z)$, defined as follows :

$$
\begin{aligned}
& \mu_{A}(n)=\frac{1}{1+n} \text { and } v_{A}(n)=\frac{n}{2+2 n}, \text { for any } n \in \mathbb{N} \\
& \mu_{B}(x)=\left\{\begin{array}{c}
0.25, \text { if } x \in[-1,1] \\
0, \text { Otherwise, }
\end{array} \text { and } v_{B}(x)=\left\{\begin{array}{c}
0.5, \text { if } x \in[-1,1] \\
1, \text { Otherwise, }
\end{array}\right.\right. \\
& \mu_{C}(x)=\frac{|\cos (x)|}{3} \text { and } v_{C}(x)=\frac{|\sin (x)|}{3}
\end{aligned}
$$

for any $x \in \mathbb{R}$.

We define an intuitionistic fuzzy mappings $f_{R}: A \rightarrow B$ and $g_{S}: B \rightarrow C$ by:

(i) an ordinary mappings $f:$ Supp $A \longrightarrow$ Supp B, defined for any $n \in$ Supp A by :

$$
\begin{aligned}
& f(n)=\left\{\begin{array}{l}
1, \text { if } n \text { is even number, } \\
-1, \text { if } n \text { odd is number, }
\end{array}\right. \\
& \text { and } g: \text { Supp } B \longrightarrow \text { Supp C defined by } g(x)=2 x, \text { for any } x \in[-1,1] .
\end{aligned}
$$

(ii) an IF-relations $R$ and $S$ defined by:

$$
\begin{aligned}
& \mu_{R}(n, f(n))=\wedge\left\{\mu_{A}(n), \mu_{B}(f(n))\right\}=\wedge\left\{\frac{1}{1+n}, 0.25\right\} \text { and } v_{R}(n, f(n))=\vee\left\{v_{A}(n), v_{B}(f(n))\right\}=\vee\left\{\frac{n}{2+2 n}, 0.5\right\} \text { and } \mu_{S}(x, g(x))= \\
& \wedge\left\{\mu_{B}(x), \mu_{C}(g(x))\right\}=\left\{\begin{array}{c}
\wedge\left\{0.25, \frac{|\cos (2 x)|}{3}\right\}, x \in[-1,1], \\
0, \text { otherwise, }
\end{array}\right. \\
& \text { and } v_{S}(x, g(x))=\vee\left\{v_{B}(x), v_{C}(g(x))\right\}=\left\{\begin{array}{r}
\left.\vee 0.5, \frac{|\sin (2 x)|}{3}\right\}, x \in[-1,1], \\
1, \text { otherwise, }
\end{array}\right.
\end{aligned}
$$

Then, the composition $g_{S} \circ f_{R}=(g \circ f)_{T}$ is defined by :

(i) an ordinary mapping $f: \operatorname{Supp} A \longrightarrow \operatorname{Supp} C$, defined for any $n \in \operatorname{Supp} A$ by :

$$
(g \circ f)(n)=\left\{\begin{array}{l}
2, \text { if } n \text { is even number, } \\
-2, \text { if } n \text { is odd number },
\end{array}\right.
$$

(ii) an IF-relation $T$ defined by:

$$
\begin{aligned}
\mu_{T}(n,(g \circ f)(n))= & \left\{\begin{array}{r}
\wedge\left\{\frac{1}{1+n}, 0.25, \frac{|\cos (2)|}{3}\right\}, \text { if nis even number } \\
\wedge\left\{\frac{1}{1+n}, 0.25, \frac{|\cos (-2)|}{3}\right\}, \text { if nis odd number }
\end{array}\right. \\
= & \wedge\left\{\frac{1}{1+n}, 0.25, \frac{|\cos (2)|}{3}\right\} \\
= & \wedge\left\{\frac{1}{1+n}, 0.25\right\}
\end{aligned}
$$




$$
\begin{aligned}
v_{T}(n,(g \circ f)(n)) & =\left\{\begin{array}{r}
\vee\left\{\frac{n}{2+2 n}, 0.25, \frac{|\sin (2)|}{3}\right\}, \text { if nis even number } \\
\vee\left\{\frac{n}{2+2 n}, 0.25, \frac{|\sin (-2)|}{3}\right\}, \text { if nis odd number }
\end{array}\right. \\
& =\vee\left\{\frac{n}{2+2 n}, 0.25, \frac{|\sin (2)|}{3}\right\} \\
& =\vee\left\{\frac{2}{2+2 n}, 0.25\right\} .
\end{aligned}
$$

Proposition 3.9. The composition of intuitionistic fuzzy mappings is an associative operation.

Proof. Let $A, B, C$ and $D$ are an intuitionistic fuzzy sets on $X, Y, Z$ and $T$ respectively, let $f_{R_{1}}: A \rightarrow B, g_{R_{2}}: B \rightarrow C$ and $h_{R_{3}}: C \rightarrow D$ are an intuitionistic fuzzy mappings. We need to show that $h_{R_{3}} \circ\left(g_{R_{2}} \circ f_{R_{1}}\right)=\left(h_{R_{3}} \circ g_{R_{2}}\right) \circ f_{R_{1}}$. On the one hand, it is easy to verify that $(h \circ(g \circ f))=((h \circ g) \circ f)$. On the one hand,

$$
\begin{aligned}
\mu_{R_{3} \circ\left(R_{2} \circ R_{1}\right)}(x, h \circ(g \circ f)(x)) & \left.=\min \left\{\mu_{R_{2} \circ R_{1}}(x, g \circ f(x)), \mu_{R_{3}}(g \circ f(x)), h \circ(g \circ f)(x)\right\}\right\} \\
& =\min \left\{\min \left\{\mu_{R_{1}}(x, f(x)), \mu_{R_{2}}(f(x), g(f(x))\}, \mu_{R_{3}}(g \circ f(x), h \circ(g \circ f)(x))\right\}\right. \\
& =\min \left\{\mu_{R_{1}}(x, f(x)), \mu_{R_{2}}\left(f(x), g(f(x)), \mu_{R_{3}}(g \circ f(x), h \circ(g \circ f)(x))\right\}\right. \\
& =\min \left\{\mu_{A}(x), \mu_{B}(f(x)), \mu_{C}\left(g(f(x)), \mu_{D}((h \circ g) \circ f)(x)\right)\right\} \\
& =\min \left\{\mu_{R_{1}}(x, f(x)), \mu_{R_{2}}\left(f(x), g(f(x)), \mu_{R_{3}}(g \circ f(x),(h \circ g) \circ f)(x)\right)\right\} \\
& =\min \left\{\mu_{R_{1}}(x, f(x)), \min \left\{\mu_{R_{2}}(f(x), g(f(x))), \mu_{R_{3}}(g \circ f(x),(h \circ(g \circ f))(x))\right\}\right\} \\
& \left.=\min \left\{\mu_{R_{1}}(x, f(x)), \mu_{R_{3} \circ R_{2}}(f(x),(g \circ f)(x))\right\}\right\} \\
& =\mu_{\left(R_{3} \circ R_{2}\right) \circ R_{1}}(x,((h \circ g) \circ f)(x))
\end{aligned}
$$

In similar way, we prove that $v_{R_{3} \circ\left(R_{2} \circ R_{1}\right)}(x, h \circ(g \circ f)(x))=v_{\left(R_{3} \circ R_{2}\right) \circ R_{1}}(x,(h \circ g) \circ f(x))$.

Remark 3.10. The intuitionistic fuzzy identity mapping $I d_{R}$ is neutral for the composition of intuitionistic fuzzy mappings.

In the sequel, we need to introduce the notion of the direct image and the inverse image of intuitionistic fuzzy set by an intuitionistic fuzzy mapping.

Definition 3.11. Let $f_{R}: A \rightarrow B$ be an intuitionistic fuzzy mapping from an intuitionistic fuzzy set $A$ to another intuitionistic fuzzy set $B$ and $C \subseteq A$. The direct image of $C$ by $f_{R}$ is defined by $f_{R}(C)=\left\{\left\langle y, \mu_{f_{R}(C)}(y), v_{f_{R}(C)}(y)\right\rangle \mid y \in Y\right\}$, where

$$
\mu_{f_{R}(C)}(y)=\left\{\begin{array}{c}
\mu_{B}(y), \text { if } y \in f(\operatorname{supp}(C)) \\
0, \text { Otherwise }
\end{array}\right.
$$

and

$$
v_{f_{R}(C)}(y)=\left\{\begin{array}{c}
v_{B}(y), \text { if } y \in f(\operatorname{supp}(C)) \\
1, \text { Otherwise }
\end{array}\right.
$$

Similarly, if $C^{\prime} \subseteq B$. The inverse image of $C^{\prime}$ by $f$ is defined by $f_{R}^{-1}\left(C^{\prime}\right)=\left\{\left\langle x, \mu_{f_{R}^{-1}\left(C^{\prime}\right)}(x), v_{f_{R}^{-1}\left(C^{\prime}\right)}(x)\right\rangle \mid x \in X\right\}$, where

$$
\mu_{f_{R}^{-1}\left(C^{\prime}\right)}(x)=\left\{\begin{array}{c}
\mu_{A}(x), \text { if } x \in f^{-1}\left(\operatorname{supp}\left(C^{\prime}\right)\right) \\
0, \text { Otherwise }
\end{array}\right.
$$

and

$$
v_{f_{R}^{-1}\left(C^{\prime}\right)}(x)=\left\{\begin{array}{c}
v_{A}(x), \text { if } x \in f^{-1}\left(\operatorname{supp}\left(C^{\prime}\right)\right) \\
1, \text { Otherwise }
\end{array}\right.
$$

Example 3.12. Let $X=\mathscr{P}(\mathbb{R}), Y=\{\alpha, \beta\}$ and $A \in I F S(X)$ defined for any $S \in \mathscr{P}(\mathbb{R})$ by :

$$
\begin{aligned}
& \mu_{A}(S)=\left\{\begin{array}{c}
0.55, \text { if } \text { Sis denumerable set } \\
0, \text { Otherwise },
\end{array}\right. \\
& v_{A}(S)=\left\{\begin{array}{c}
0.3, \text { if } \text { Sis denumerable set } \\
1, \text { Otherwise. }
\end{array}\right.
\end{aligned}
$$

Also, let $B \in I F S(Y)$ given by $B=\{\langle\alpha, 0.2,0.5\rangle,\langle\beta, 0.7,0.3\rangle\}$.

We define the intuitionistic fuzzy mapping $f_{R}: A \rightarrow B$ by:

(i) an ordinary mapping $f: \operatorname{Supp} A \longrightarrow \operatorname{Supp} B$, defined for any $S \in$ Supp A by

$$
f(S)=\left\{\begin{array}{c}
\alpha, \text { if Sis finite set } \\
\beta, \text { Otherwise, }
\end{array}\right.
$$

(ii) an IF-relation $R$ defined by $\mu_{R}(S, f(S))=\mu_{A}(S) \wedge \mu_{B}(f(S))=0.55 \wedge 0.2=0.2$ and $v_{R}(S, f(S))=v_{A}(S) \vee v_{B}(f(S))=0.3 \vee 0.5=0.5$ 
Now, if we take $C$ an $I F$-set on $X$, where $C \subseteq A$ given by:

$$
\begin{aligned}
& \mu_{C}(S)=\left\{\begin{array}{c}
0.4, \text { if } \text { Sis finite set } \\
0, \text { Otherwise },
\end{array}\right. \\
& v_{C}(S)=\left\{\begin{array}{c}
0.4, \text { if } \text { Sis finite set } \\
1, \text { Otherwise. }
\end{array}\right.
\end{aligned}
$$

Then, the direct image of $C$ by $f_{R}$ is defined by:

$\mu_{f_{R}(C)}(y)=\left\{\begin{array}{c}\mu_{B}(y), \text { if } y \in f(\operatorname{supp}(C)) \\ 0, \text { Otherwise, }\end{array} \quad=\left\{\begin{array}{c}0.2, \text { if } y=\alpha \\ 0, \text { if } y=\beta\end{array}\right.\right.$

and

$$
v_{f_{R}(C)}(y)=\left\{\begin{array}{c}
\mu_{B}(y), \text { if } y \in f(\operatorname{supp}(C)) \\
0, \text { Otherwise, }
\end{array} \quad=\left\{\begin{array}{c}
0.5, \text { if } y=\alpha \\
1, \text { if } y=\beta .
\end{array}\right.\right.
$$

Moreover, it is easy to show that $f_{R}(C) \subseteq B$.

Next, if we take $C^{\prime}$ an IF-set on $Y$, where $C^{\prime} \subseteq B$ given by:

$$
\mu_{C^{\prime}}(y)=\left\{\begin{array}{c}
0.1, \text { if } y=\alpha \\
0, y=\beta,
\end{array} \quad \text { and } v_{C^{\prime}}(y)=\left\{\begin{array}{c}
0.6, \text { if } y=\alpha \\
1, y=\beta .
\end{array}\right.\right.
$$

Then, the inverse image of $C^{\prime}$ by $f$ is defined by:

$\mu_{f_{R}^{-1}\left(C^{\prime}\right)}(S)=\left\{\begin{array}{c}\mu_{A}(S), \text { if } S \in f^{-1}\left(\operatorname{supp}\left(C^{\prime}\right)\right) \\ 0, \text { Otherwise, }\end{array} \quad=\left\{\begin{array}{c}0.55, \text { if } S \text { is finite set } \\ 0, \text { Otherwise }\end{array}\right.\right.$

and

$v_{f_{R}^{-1}\left(C^{\prime}\right)}(S)=\left\{\begin{array}{c}v_{A}(S), \text { if } S \in f^{-1}\left(\operatorname{supp}\left(C^{\prime}\right)\right) \\ 0, \text { Otherwise }\end{array} \quad=\left\{\begin{array}{c}0.3, \text { if } S \text { is finite set } \\ 1, \text { Otherwise } .\end{array}\right.\right.$

Moreover, it is easy to show that $f_{R}^{-1}\left(C^{\prime}\right) \varsubsetneqq A$ in the case of $S=\mathbb{N}$.

Definition 3.13. Let $A$ be an intuitionistic fuzzy set on a set $X$ and $\sim$ be an equivalence relation over Supp $(A)$, let $B$ an intuitionistic fuzzy set on $\mathscr{P}(X)$ defined by :

$$
\mu_{B}(\theta)=\left\{\begin{array}{c}
\mu_{A}(x), \text { if } \theta=\bar{x} \in \operatorname{supp}(A) / \sim \\
0, \text { Otherwise }
\end{array}\right.
$$

and

$$
v_{B}(\theta)=\left\{\begin{array}{c}
v_{A}(x), \text { if } \theta=\bar{x} \in \operatorname{supp}(A) / \sim \\
1, \text { Otherwise. }
\end{array}\right.
$$

Then, the intuitionistic fuzzy mapping $P_{R}: A \longrightarrow B$ defined by :

(i) an ordinary mapping $P: \operatorname{Supp}(A) \longrightarrow \operatorname{Supp}(B)$ such that $P(x)=\bar{x}$ for any $x \in \operatorname{Supp}(A)$,

(ii) an intuitionistic fuzzy relation $R$ defined by:

$$
\begin{aligned}
\mu_{R}(x, P(x)) & =\min \left\{\mu_{A}(x), \mu_{B}(P(x))\right\} \\
& =\min \left\{\mu_{A}(x), \mu_{B}(\bar{x})\right\} \\
& =\min \left\{\mu_{A}(x), \mu_{A}(x)\right\} \\
& =\mu_{A}(x)
\end{aligned}
$$

and

$$
\begin{aligned}
v_{R}(x, P(x)) & =\max \left\{v_{A}(x), v_{B}(P(x))\right\} \\
& =\max \left\{v_{A}(x), v_{B}(\bar{x})\right\} \\
& =\max \left\{v_{A}(x), v_{A}(x)\right\} \\
& =v_{A}(x)
\end{aligned}
$$

is called the intuitionistic fuzzy projection mapping.

Now, we define the product of intuitionistic fuzzy sets and intuitionistic fuzzy projection mappings.

Definition 3.14. Let $A$ be an intuitionistic fuzzy set on $X$ and $B$ be an intuitionistic fuzzy set on $Y$. The product of $A$ and $B$, denoted by $A \times B$ is an intuitionistic fuzzy set on $X \times Y$ defined by:

$\mu_{X \times Y}(x, y)=\min \left\{\mu_{A}(x), \mu_{B}(y)\right\}$ and $v_{X \times Y}(x, y)=\max \left\{v_{A}(x), v_{B}(y)\right\}$.

Also, we define the first intuitionistic fuzzy projection mapping $\left(P_{1}\right)_{R}: A \times B \longrightarrow A$ by:

(i) an ordinary mapping $P_{1}: \operatorname{Supp}(A \times B) \longrightarrow \operatorname{Supp}(A)$ such that $P_{1}(x, y)=x$ for any $(x, y) \in \operatorname{Supp}(A \times B)$, 
(i) an intuitionistic fuzzy relation $R$ defined by :

$$
\begin{aligned}
\mu_{R}\left((x, y), P_{1}(x, y)\right) & =\min \left\{\mu_{A \times B}(x, y), \mu_{A}\left(P_{1}(x, y)\right)\right\} \\
& =\min \left\{\mu_{A}(x), \mu_{B}(y), \mu_{A}(x)\right\} \\
& =\min \left\{\mu_{A}(x), \mu_{B}(y)\right\}
\end{aligned}
$$

and

$$
\begin{aligned}
v_{R}\left((x, y), P_{1}(x, y)\right) & =\max \left\{v_{A \times B}(x, y), v_{A}\left(P_{1}(x, y)\right)\right\} \\
& =\max \left\{v_{A}(x), v_{B}(y), v_{A}(x)\right\} \\
& =\max \left\{v_{A}(x), v_{B}(y)\right\}
\end{aligned}
$$

The second intuitionistic fuzzy projection mapping is defined analogously.

Next, we introduce the notion of disjoint union of intuitionistic fuzzy sets and intuitionistic fuzzy inclusion mappings.

Definition 3.15. Let $A$ be an intuitionistic fuzzy set on $X$ and $B$ be an intuitionistic fuzzy set on $Y$. The disjoint union of $A$ and $B$, denoted by $A \sqcup B$ is an intuitionistic fuzzy set on $X \times\{1\} \cup Y \times\{2\}$ defined by :

$$
\mu_{A \sqcup B}(x, k)=\left\{\begin{array}{l}
\mu_{A}(x), \text { if } k=1 \\
\mu_{B}(x), \text { if } k=2
\end{array}\right.
$$

and

$$
v_{A \sqcup B}(x, k)=\left\{\begin{array}{l}
v_{A}(x), \text { if } k=1 \\
v_{B}(x), \text { if } k=2
\end{array}\right.
$$

Also, we define the first intuitionistic fuzzy inclusion mapping $\left(\varphi_{1}\right)_{R}: A \longrightarrow A \sqcup B$ by :

(i) an ordinary mapping $\varphi_{1}$, defined by:

$$
\varphi_{1}: \operatorname{Supp}(A) \longrightarrow \operatorname{Supp}(A \sqcup B) \text { such that } \varphi_{1}(x)=(x, 1) \text { for any } x \in \operatorname{Supp}(A),
$$

(ii) an intuitionistic fuzzy relation $R$ defined by:

$$
\begin{aligned}
\mu_{R}\left(x, \varphi_{1}(x)\right) & =\min \left\{\mu_{A}(x), \mu_{A \sqcup B}\left(\varphi_{1}(x)\right)\right\} \\
& =\min \left\{\mu_{A}(x), \mu_{A \sqcup B}(x, 1)\right\} \\
& =\min \left\{\mu_{A}(x), \mu_{A}(x)\right\} \\
& =\mu_{A}(x)
\end{aligned}
$$

and

$$
\begin{aligned}
v_{R}\left(x, \varphi_{1}(x)\right) & =\max \left\{v_{A}(x), v_{A \sqcup B}\left(\varphi_{1}(x)\right)\right\} \\
& =\max \left\{v_{A}(x), v_{A \sqcup B}(x, 1)\right\} \\
& =\max \left\{v_{A}(x), v_{A}(x)\right\} \\
& =v_{A}(x)
\end{aligned}
$$

The second intuitionistic fuzzy inclusion mapping is defined analogously.

\section{Applications}

In this section, we establish as an application the intuitionistic fuzzy continuous mapping in intuitionistic fuzzy topological spaces.

\subsection{Intuitionistic fuzzy topology}

This subsection is devoted to study the structure of intuitionistic fuzzy topology as a generalization of the structure of fuzzy topology given by Kandil et al. [17].

Definition 4.1. Let $A$ be an intuitionistic fuzzy set on the set $X$ and $O_{A}=\{U$ is an IFS on $X: U \subseteq A\}$. We define an intuitionistic fuzzy topology on intuitionistic fuzzy set $A$ by the family $T \subseteq O_{A}$ which satisfies the following conditions :

(i) $A, 0_{\sim} \in T$;

(ii) if $U_{1}, U_{2} \in T$, then $U_{1} \cap U_{2} \in T$;

(iii) if $U_{i} \in T$ for all $i \in I$, then $\cup_{I} U_{i} \in T$.

$T$ is called an intuitionistic fuzzy topology of $A$ and the pair $(A, T)$ is an intuitionistic fuzzy topological space (IF-TOP, for short). Every element of $T$ is called an intuitionistic fuzzy open set (IFOS, for short). 
Example 4.2. Let $X$ be a nonempty set and $A$ be an intuitionistic fuzzy set on $\mathscr{P}(X)$ given by : $\mu_{A}(\theta)=\left\{\begin{array}{c}1, \text { if } \theta=\emptyset \\ 0.5,0<|\theta|<\infty, \\ 0, \text { Otherwise, }\end{array}\right.$ and $v_{A}(\theta)=\left\{\begin{array}{c}0, \text { if } \theta=\emptyset \\ 0.4,0<|\theta|<\infty, \\ 0.2, \text { Otherwise, }\end{array}\right.$

Then, the family $T=\left\{A, 0_{\sim}, U\right\}$ where:

$\mu_{U}(\theta)=\left\{\begin{array}{c}0.4,|\theta|<\infty, \\ 0, \text { Otherwise, }\end{array} \quad\right.$ and $v_{U}(\theta)=\left\{\begin{array}{c}0.6,|\theta|<\infty, \\ 0.5, \text { Otherwise, }\end{array}\right.$

is an intuitioniste fuzzy topology on A.

Inspired by the notion of interior (resp. closure) on intuitionistic fuzzy topological space on a set introduced by Atanassov [3], authors define these notions in intuitionistic fuzzy topology on an intuitionistic fuzzy set.

Definition 4.3. Let $(A, T)$ be an intuitionistic fuzzy topological space, for every intuitionistic fuzzy subset $G$ of $X$ we define the interior and closure of $G$ by:

$\operatorname{int}(G)=\left\{\left\langle x, \max _{x \in X} \mu_{U}(x), \min _{x \in X} v_{U}(x)\right\rangle \mid x \in U \subseteq G\right\}$

and

$\operatorname{cl}(G)=\left\{\left\langle x, \min _{x \in X} \mu_{K}(x), \max _{x \in X} v_{K}(x)\right\rangle \mid x \in A\right.$ and $\left.G \subseteq K\right\}$

Example 4.4. Let $X=\{a, b, c\}$ and $A, B, C, D \in I F S(X)$ such that

$A=\{\langle a, 0.5,0.1\rangle,\langle b, 0.7,0.2\rangle,\langle c, 0.6,0\rangle\}$

$B=\{\langle a, 0.5,0.2\rangle,\langle b, 0.5,0.4\rangle,<c, 0.4,0.4\rangle\}$

$C=\{\langle a, 0.4,0.5\rangle,\langle b, 0.6,0.3\rangle,<c, 0.2,0.3\rangle\}$

$D=\{\langle a, 0.5,0.2\rangle,\langle b, 0.6,0.3\rangle,<c, 0.4,0.3\rangle\}$

$E=\{<a, 0.4,0.5\rangle,\langle b, 0.5,0.4\rangle,<c, 0.2,0.4\rangle\}$

Then the family $T=\left\{A, 0_{\sim}, B, C, D, E\right\}$ is an IFT of $A$.

Now, we suppose that $G \in \operatorname{IFS}(X)$ given by $G=\{<a, 0.41,0.49),<b, 0.61,0.29>,<c, 0.2,0.2>\}$. Then, int $(G)=C \cup E=C$ and $\operatorname{cl}(G)=1 \sim$.

Definition 4.5. [9] Let $(A, T)$ be an intuitionistic fuzzy topological space and $U \in I F S(A, T)$. Then $U$ is called :

1. an intuitionistic fuzzy semiopen set (IFSOS) if $U \subseteq \operatorname{cl}(\operatorname{int}(U))$;

2. an intuitionistic fuzzy $\alpha$-open set (IF $\alpha O S)$ if $U \subseteq \operatorname{int}(\operatorname{cl}(\operatorname{int}(U)))$;

3. an intuitionistic fuzzy preopen set (IFPOS) if $U \subseteq \operatorname{int}(\operatorname{cl}(U))$;

4. an intuitionistic fuzzy regular open set (IFROS) if $U=\operatorname{int}(\mathrm{cl}(U))$.

\subsection{Intuitionistic fuzzy continuous mappings}

The present section contains an interesting properties of intuitionistic fuzzy continuous mappings in intuitionistic fuzzy topological space and relations between various kinds of intuitionistic fuzzy continuous mapping. First, the notion of intuitionistic fuzzy continuous mapping is introduced.

Definition 4.6. Let $(A, T)(B, L)$ be two intuitionistic fuzzy topological spaces. The mapping $f_{R}:(A, T) \rightarrow(B, L)$ is an intuitionistic fuzzy continuous if and only if the inverse of each $L$-open intuitionistic fuzzy set is $T$-open intuitionistic fuzzy set.

Example 4.7. Let $(A, T)$ and $\left(B, T^{\prime}\right)$ be two intuitionistic fuzzy topologies, where

$\mu_{A}(x)=0.55$ and $v_{A}(x)=0.4$, for any $x \in \mathbb{R}$ and

$\mu_{B}(y)=\left\{\begin{array}{c}0.5, \text { if } y \geq 0 \\ 0.8, \text { Otherwise, }\end{array}\right.$

and

$v_{B}(y)=\left\{\begin{array}{c}0.2, \text { if } y \geq 0 \\ 0.1, \text { Otherwise, }\end{array}\right.$

We suppose that $T=\left\{A, 0_{\sim}, U_{1}\right\}$, where

$\mu_{U_{1}}(x)=\left\{\begin{array}{c}0.55, \text { if } x \in \mathbb{R} \backslash[-2,0] \\ 0, \text { Otherwise, }\end{array} \quad\right.$ and $\quad v_{U_{1}}(x)=\left\{\begin{array}{c}0.4, \text { if } x \in \mathbb{R} \backslash[-2,0] \\ 1, \text { Otherwise, }\end{array}\right.$

Also, we suppose that $T^{\prime}=\left\{B, 0_{\sim}, U_{1}^{\prime}\right\}$, where

$\mu_{U_{1}^{\prime}}(y)=\left\{\begin{array}{l}0.5, \text { if } y \geq 0 \\ 0, \text { Otherwise, }\end{array} \quad\right.$ and $\quad v_{U_{1}^{\prime}}(y)=\left\{\begin{array}{l}0.3, \text { if } y \geq 0 \\ 1, \text { Otherwise. }\end{array}\right.$

Then, the intuitionistic fuzzy mapping $f_{R}: A \rightarrow B$ define by :

(i) an ordinary mapping $f: \mathbb{R} \longrightarrow \mathbb{R}$ such that $f(x)=(x+1)^{2}-1$ for any $x \in \mathbb{R}$, 
(i) an intuitionistic fuzzy relation $R$ defined by:

$$
\mu_{R}(x, f(x))=\left\{\begin{array}{c}
0.5, \text { if } x \in \mathbb{R} \backslash[-2,0] \\
0.55, \text { Otherwise, }
\end{array} \quad \text { and } v_{R}(x, f(x))=0.4 .\right.
$$

is an intuitionistic fuzzy continuous mapping.

Indeed, it is easy to show that $f_{R}^{-1}(B)=A$ and $f_{R}^{-1}\left(0_{\sim}\right)=0_{\sim}$ and we have,

$$
\begin{aligned}
\mu_{f_{R}^{-1}\left(U_{1}^{\prime}\right)}(x) & =\left\{\begin{array}{c}
\mu_{A}(x), \text { if } x \in f^{-1}\left(\operatorname{supp}\left(U_{1}^{\prime}\right)\right) \\
0, \text { Otherwise },
\end{array}\right. \\
& =\left\{\begin{array}{c}
\mu_{A}(x), \text { if } x \in \mathbb{R} \backslash[-2,0] \\
0, \text { Otherwise },
\end{array}\right. \\
& =\mu_{U_{1}}(x),
\end{aligned}
$$

and

$$
\begin{aligned}
v_{f_{R}^{-1}\left(U_{1}^{\prime}\right)}(x) & =\left\{\begin{array}{c}
v_{A}(x), \text { if } x \in f^{-1}\left(\operatorname{supp}\left(U_{1}^{\prime}\right)\right) \\
1, \text { Otherwise },
\end{array}\right. \\
& =\left\{\begin{array}{c}
v_{A}(x), \text { if } x \in \mathbb{R} \backslash[-2,0] \\
1, \text { Otherwise },
\end{array}\right. \\
& =\left\{\begin{array}{c}
0.4, \text { if } x \in \mathbb{R} \backslash[-2,0] \\
1, \text { Otherwise },
\end{array}\right. \\
& =v_{U_{1}}(x) .
\end{aligned}
$$

Hence, $f_{R}^{-1}\left(U_{1}^{\prime}\right)=U_{1} \in T$. Thus, $f_{R}$ is an intuitionistic fuzzy continuous mapping.

Remark 4.8. Let $(A, T)$ be an intuitionistic fuzzy topological space. Then the intuitionistic fuzzy identity mapping $\operatorname{Id}_{R}:(A, T) \rightarrow(A, T)$ is an intuitionistic fuzzy continuous mapping.

Next, the relations between various kinds of intuitionistic fuzzy continuous mapping are provided. First, the definitions of precontinuous mapping, $\alpha$-continuous mapping introduced by Gürçay et al. [9] need to be recalled.

Definition 4.9. [9] Let $f_{R}:(A, T) \rightarrow\left(B, T^{\prime}\right)$ be an intuitionistic fuzzy mapping. Then $f_{R}$ is called :

1. an intuitionistic fuzzy precontinuous mapping if $f_{R}^{-1}\left(U^{\prime}\right)$ is an IFPOS on A for every IFOS $U^{\prime}$ on $B$;

2. an intuitionistic fuzzy $\alpha$-continuous mapping if $f_{R}^{-1}\left(U^{\prime}\right)$ is an IF $\alpha O S$ on A for every IFOS $U^{\prime}$ on $B$.

The following proposition shows the relationship between intuitionistic fuzzy continuous mapping and intuitionistic fuzzy $\alpha$-continuous mapping.

Proposition 4.10. Let $f_{R}:(A, T) \rightarrow\left(B, T^{\prime}\right)$ be an intuitionistic fuzzy mapping. If $f_{R}$ is an intuitionistic fuzzy continuous mapping, then $f_{R}$ is an intuitionistic fuzzy $\alpha$-continuous mapping.

Proof. Let $U^{\prime}$ be an IFOS in $B$ and we need to show that $f_{R}^{-1}\left(U^{\prime}\right)$ is an IF $\alpha \mathrm{OS}$ in $A$. The fact that $f_{R}$ is an intuitionistic fuzzy continuous mapping implies that $f_{R}^{-1}\left(U^{\prime}\right)$ is an IFOS in $A$. From Definition 3.11, it follows that

$\mu_{f_{R}^{-1}\left(U^{\prime}\right)}(x)=\left\{\begin{array}{c}\mu_{A}(x), \text { if } x \in f^{-1}\left(\operatorname{supp}\left(U^{\prime}\right)\right) \\ 0, \text { Otherwise }\end{array}\right.$

and

$v_{f_{R}^{-1}\left(U^{\prime}\right)}(x)=\left\{\begin{array}{c}v_{A}(x), \text { if } x \in f^{-1}\left(\operatorname{supp}\left(U^{\prime}\right)\right) \\ 1, \text { Otherwise. }\end{array}\right.$

We conclude that, $f_{R}^{-1}\left(U^{\prime}\right)$ is an IF $\alpha \mathrm{OS}$ in $A$. Hence, $f_{R}$ is an intuitionistic fuzzy $\alpha$-continuous mapping.

Remark 4.11. The converse of the above implication does not necessarily hold. Indeed, let us consider the intuitionistic fuzzy mapping $f_{R}$ given in Example 4.7 and $T^{\prime}$ be an IF-topology given by $T^{\prime}=\left\{0_{\sim}, B, U_{2}^{\prime}\right\}$, where:

$\mu_{U_{2}^{\prime}}(y)=\left\{\begin{array}{c}0.3, \text { if } y \geq-\frac{1}{2} \\ 0, \text { Otherwise, }\end{array} \quad\right.$ and $v_{U_{2}^{\prime}}(y)=\left\{\begin{array}{c}0.4, \text { if } y \geq-\frac{1}{2} \\ 1, \text { Otherwise. }\end{array}\right.$

It is easy to verify that

$\mu_{f_{R}^{-1}\left(U_{2}^{\prime}\right)}(x)=\left\{\begin{array}{c}\mu_{A}(x), \text { if } x \in f^{-1}\left(\operatorname{supp}\left(U_{2}^{\prime}\right)\right) \\ 0, \text { Otherwise, }\end{array} \quad=\left\{\begin{array}{c}0.55, \text { if } x \in \mathbb{R} \backslash\left[-\frac{\sqrt{2}}{2}-1, \frac{\sqrt{2}}{2}-1\right] \\ 0, \text { Otherwise, }\end{array}\right.\right.$

and

$v_{f_{R}^{-1}\left(U_{2}^{\prime}\right)}(x)=\left\{\begin{array}{c}v_{A}(x), \text { if } x \in f^{-1}\left(\operatorname{supp}\left(U_{2}^{\prime}\right)\right) \\ 1, \text { Otherwise, }\end{array}=\left\{\begin{array}{c}0.4, \text { if } x \in \mathbb{R} \backslash\left[-\frac{\sqrt{2}}{2}-1, \frac{\sqrt{2}}{2}-1\right] \\ 1, \text { Otherwise, }\end{array}\right.\right.$

Hence, $\operatorname{int}\left(f_{R}^{-1}\left(U_{2}^{\prime}\right)\right)=U_{1}$ and $\operatorname{cl}\left(U_{1}\right)=1_{\sim}$ and $\operatorname{int}\left(1_{\sim}\right)=A$. Thus, $f_{R}^{-1}\left(U_{2}^{\prime}\right) \subseteq \operatorname{int}\left(\operatorname{cl}\left(\operatorname{int}\left(f_{R}^{-1}\left(U_{2}^{\prime}\right)\right)\right)\right.$. We conclude that $f_{R}^{-1}\left(U_{2}^{\prime}\right)$ is an IF $\alpha$ S but not IFOS and $f_{R}$ is an intuitionistic fuzzy $\alpha$-continuous but not an intuitionistic fuzzy continuous. 
The following proposition shows the relationship between intuitionistic fuzzy $\alpha$-continuous mapping and intuitionistic fuzzy pre-continuous mapping.

Proposition 4.12. Let $f_{R}:(A, T) \rightarrow\left(B, T^{\prime}\right)$ be an intuitionistic fuzzy mapping. If $f_{R}$ is an intuitionistic fuzzy $\alpha$-continuous mapping, then $f_{R}$ is an intuitionistic fuzzy pre-continuous mapping.

Proof. Let $U^{\prime}$ be an IFOS in $B$ and we need to show that $f_{R}^{-1}\left(U^{\prime}\right)$ is an IFPOS in $A$. The fact that $f_{R}$ is an intuitionistic fuzzy $\alpha$-continuous mapping implies that $f_{R}^{-1}\left(U^{\prime}\right)$ is an IF $\alpha \mathrm{OS}$ in $A$. From Definition 3.11, it follows that

$\mu_{f_{R}^{-1}\left(U^{\prime}\right)}(x)=\left\{\begin{array}{c}\mu_{A}(x), \text { if } x \in f^{-1}\left(\operatorname{supp}\left(U^{\prime}\right)\right) \\ 0, \text { Otherwise, }\end{array}\right.$
$v_{f_{R}^{-1}\left(U^{\prime}\right)}(x)=\left\{\begin{array}{c}v_{A}(x), \text { if } x \in f^{-1}\left(\operatorname{supp}\left(U^{\prime}\right)\right) \\ 1, \text { Otherwise, }\end{array}\right.$

We conclude that, $f_{R}^{-1}\left(U^{\prime}\right)$ is an IFPOS in $A$. Hence, $f_{R}$ is an intuitionistic fuzzy pre-continuous mapping.

Remark 4.13. The converse of the above implication is not necessarily holds. Indeed, let us consider the intuitionistic fuzzy mapping $f_{R}$ given in Example 4.7 and $T^{\prime}$ be an IF-topology given by $T^{\prime}=\left\{0_{\sim}, B, U_{3}^{\prime}\right\}$, where:

$\mu_{U_{3}^{\prime}}(y)=\left\{\begin{array}{c}0.3, \text { if } y \in[-1,0] \\ 0, \text { Otherwise, }\end{array} \quad\right.$ and $v_{U_{3}^{\prime}}(y)=\left\{\begin{array}{r}0.4, \text { if } y \in[-1,0] \\ 1, \text { Otherwise. }\end{array}\right.$

It is easy to verify that

$\mu_{f_{R}^{-1}\left(U_{3}^{\prime}\right)}(x)=\left\{\begin{array}{c}\mu_{A}(x), \text { if } x \in f^{-1}\left(\operatorname{supp}\left(U_{3}^{\prime}\right)\right) \\ 0, \text { Otherwise, }\end{array} \quad=\left\{\begin{array}{c}0.55, \text { if } x \in[-2,0] \\ 0, \text { Otherwise }\end{array}\right.\right.$

and

$v_{f_{R}^{-1}\left(U_{3}^{\prime}\right)}(x)=\left\{\begin{array}{c}v_{A}(x), \text { if } x \in[-2,0] \\ 1, \text { Otherwise, }\end{array} \quad=\left\{\begin{array}{c}0.4, \text { if } x \in[-2,0] \\ 1, \text { Otherwise. }\end{array}\right.\right.$

Hence, $\operatorname{cl}\left(f_{R}^{-1}\left(U_{3}^{\prime}\right)\right)=1_{\sim}$ and $\operatorname{int}\left(1_{\sim}\right)=A$. Thus, $f_{R}^{-1}\left(U_{3}^{\prime}\right) \subseteq \operatorname{int}\left(\operatorname{cl}\left(f_{R}^{-1}\left(U_{3}^{\prime}\right)\right)\right)$. We conclude that $f_{R}^{-1}\left(U_{3}^{\prime}\right)$ is an IFPOS and $f_{R}$ is an intuitionistic fuzzy pre-continuous but not an intuitionistic fuzzy $\alpha$-continuous.

\section{Conclusion}

In this work, the notion of intuitionistic fuzzy mapping based on the intuitionistic fuzzy relation as a generalization of the notion of fuzzy mapping defined by Ismail and Massa'deh is introduced and the most interesting properties are investigated. As applications, some properties of intuitionistic fuzzy continuous mappings in intuitionistic fuzzy topological space are provided and relations among various kinds of intuitionistic fuzzy continuity are investigated.

Future work is anticipated in multiple directions. We think it makes sense to study the notion of intuitionistic fuzzy mapping for other types of topologies based on the intuitionistic fuzzy sets. Moreover, we intend to extend this work to other kinds of intuitionistic fuzzy continuous mappings.

\section{References}

[1] K. Atanassov, Intuitionistic fuzzy sets, VII ITKRs Scientific Session, Sofia, 1983.

[2] K. Atanassov, Intuitionistic fuzzy sets, Fuzzy Sets and Systems 20 (1986), 87-96, doi:10.1016/S0165-0114(86)80034-3

[3] K. Atanassov, Intuitionistic Fuzzy Sets: Theory and Applications, Springer-Verlag, Heidelberg, New York, 1999, doi:10.1007/978-3-7908-1870-3-1

[4] P. Burillo and H. Bustince, Intuitionistic fuzzy relations (Part I), Mathware and computing 2, (1995), 5-38.

[5] P. Burillo and H. Bustince, Intuitionistic fuzzy relations (Part II), Mathware and computing 2, (1995), $117-148$.

[6] H. Bustince and P. Burillo, Antisymmetrical intuitionistic fuzzy relation. Order on the referential set induced by an bi fuzzy relation, Fuzzy Sets and Systems, Fuzzy Sets and Systems, 2 (1995), 17-22.

[7] H. Bustince and P. Burillo, Structures on intuitionistic fuzzy relations , Fuzzy Sets and Systems, 3 (1996), 293-303, doi:10.1016/0165-0114(96)84610-0

[8] P. Burillo, Construction of intuitionistic fuzzy relations with predetermined properties, Fuzzy Sets and Systems, Fuzzy Sets and Systems 3 (2000), 379-403, doi:10.1016/S0165-0114(97)00381-3

[9] H. Gürçay, D. Çoker and A.H. Eş, On fuzzy continuity in intuitionistic fuzzy topological spaces, Journal of Fuzzy Mathematics, 2 (2003), $365-378$.

[10] I. Farhan and O.M. Mourad, A new structure and constructions of L-fuzzy maps, International Journal of Computational and Applied Mathematics 1 (2013), 1-10.

[11] O. Galor, Discrete dynamical systems, Springer, 2007, doi:10.1007/3-540-36776-4

[12] G. Gomathi and D. Jayanthi, Intuitionistic fuzzy $b^{\sharp}$ continuous mapping, Advances in Fuzzy Mathematics 1 (2018), 39-47.

[13] S. Heilpern, Fuzzy mappings and fixed point theorem, Journal of Mathematical Analysis and Applications, Journal of Mathematical Analysis and Applications, 83 (1981), 566-569, doi:10.1016/0022-247X(81)90141-4

[14] G.E. Hughes and M.J. Cresswell, A New Introduction to Modal Logic, London: Routledge, (2012), doi:10.4324/9780203028100

[15] K. Hur, S.Y. Jang and H.M. Kang, Intuitionistic fuzzy subgroupoids, International Journal of Fuzzy Logic and Intelligent Systems, 1 (2003), 72-77, doi:10.5391/IJFIS.2003.3.1.072

[16] D. Jayanthi, Intuitionistic Fuzzy Generalized Beta Continuous, Indian Journal of Applied Research, Mappings, 4 (2014), 1-6.

[17] A. Kandil, S. Saleh and M.M. Yakout, Fuzzy topology on fuzzy sets: regularity and separation axioms, American Academic and Scholarly Research Journal, 2 (2012).

[18] H.W. Kang, J-G. Lee and K. Hur, Intuitionistic fuzzy mappings and intuitionistic fuzzy equivalence relations, Annals of Fuzzy Mathematics and Informatics, 1 (2012), 61-87.

[19] K. Lim, G.H. Choi and H. Hur, Fuzzy mappings and fuzzy equivalence relations, International Journal of Fuzzy Logic and Intelligent Systems, 3 (2011), 750-749, doi:10.5391/IJFIS.2011.11.3.153

[20] A. Manimaran, K. A. Prakash, P. Thangaraj, Intuitionistic fuzzy totally continuous and totally semi-continuous mappings in intuitionistic fuzzy topological spaces, International journal of Advanced Scientific and Technical Research, 2 (2011), 505-509.

[21] S.K. Sardar, M. Mandal and S.K. Majumder, Intuitionistic fuzzy ideal extensions in semigroups, J. Pure Appl. Math, 1 (2015), $59-67$. 
[22] Y. Shen, F. Wang and W. Chen, A note on intuitionistic fuzzy mappings, Iranian Journal of Fuzzy Systems, 5 (2012), 63-76.

[23] H. Simmons, An introduction to category theory, Cambridge University Press, Cambridge, New York Rydeheard DE, Burstall.

[24] L.A. Zadeh, Fuzzy sets, Information and Control, 8 (1965), 331-352, doi:10.1016/S0019-9958(65)90241-X

[25] L. Zedam, S. Milles and E. Rak, The fixed point property for intuitionistic fuzzy lattices, Fuzzy Information and Engineering, 9 (2017), 359-380, doi:10.1016/j.fiae.2017.09.007 